\title{
Exoplanet Searches by Future Deep Space Missions
}

\author{
C. Maccone ${ }^{1}$ \\ ${ }^{1}$ International Academy of Astronautics, Via Martorelli 43, 10155 Torino, Italy \\ [clmaccon@libero.it]
}

\begin{abstract}
The search for exoplanets could benefit from gravitational lensing if we could get to $550 \mathrm{AU}$ from the Sun and beyond. This is because the gravitational lens of the Sun would highly intensify there any weak electromagnetic wave reaching the solar system from distant planets in the Galaxy (see Maccone 2009). The gravitational lens of the Sun, however, has a drawback: the solar Corona. Electrons in the Corona make electromagnetic waves diverge and this pushes the focus out to distances higher than 550 AU. Jupiter is the second larger mass in the solar system after the Sun, but in this focal game not only the mass matters: rather, what really matters is the ratio between the radius of the body squared and the mass of the body. In this regard, Jupiter qualifies as the second best choice for a space mission, requiring the spacecraft to reach 6,077 AU. In this paper, we study the benefit of exoplanet searches by deep space missions.
\end{abstract}

\section{Introduction}

Einsteins deflection formula $\alpha(r)=4 G M_{\odot} / c^{2} r$ (for rays passing at distance $r$ from the Sun center) shows that largest light deflection angle $\alpha$ occurs just for those rays just grazing the Sun surface. The minimal focal distance $d_{F o c a l}$ is related to the tangent of the above maximum deflection angle by the formula $c^{2} R_{\odot}^{2} / 4 G M_{\odot}=550 \mathrm{AU}$. This is the minimal focal distance of the gravitational lens of the Sun, i.e. the minimal distance from the Sun's center that the spacecraft must reach to get magnified radio pictures of whatever lies on the other side of the Sun with respect to the spacecraft position.

\section{The huge gain provided by the Sun's gravity lens}

Having determined the minimal distance of 550 AU that the focal spacecraft must reach, one now wonders what's the good of going so far out of the solar system, i.e. how much focussing of light rays is caused by the gravitational field of the Sun. The answer to such a question is provided by the technical notion of "antenna gain", stemming out of antenna theory. In Maccone (2009), it is proven that the antenna gain of the gravitational lens of the Sun, at a given observing wavelength $\lambda$ is $G_{\odot}=4 \pi^{2} r_{\text {Schwarzschild }} / \lambda=8 \pi^{2} G M_{\odot} / \lambda c^{2}$.

Numerically, the resulting values for the gain are huge: for instance, at the water maser frequency of $22 \mathrm{GHz}$, the gain is about $70 \mathrm{~dB}$. Furthermore, F. D. Drake (personal communication) wondered whether the above equation is actually just the Suns voltage gain, and not the full Suns power gain that really matters in telecommunications. 
According to Drake, the true power gain formula for the Sun would be proportional to the Schwarzschild radius of the Sun squared over the wavelength squared, yielding a much larger value still for the Suns gain. In other words, the equation for the Suns gain would be an underestimate of the true gain value. The question is open for research in astrophysics.

\section{How electrons in the Sun's Corona "push the focus out"}

The electrons in the Suns Corona push the true focus out i.e. they create a diverging lens effect that opposes the converging lens effect of gravity. Thus, the true minimal focal distance, that the focal spacecraft must reach, is higher for lower frequencies of the sources electromagnetic waves crossing the Corona, and lower for higher frequencies. For instance, at $500 \mathrm{GHz}$ the true focus falls at about $650 \mathrm{AU}$, while at $160 \mathrm{GHz}$ (the cosmic microwave background peak frequency) the true focus is found at $763 \mathrm{AU}$, and finally at $60 \mathrm{GHz}$ the true focus is located at 1,000 AU or beyond. Thus, we might briefly say that the spacecraft must actually get beyond $550 \mathrm{AU}$ to get rid of the problems caused by the Suns Corona. Also, it must be stressed that these numbers were obtained on the basis of the so-called Baumbach-Allen model for the Solar Corona (described in Maconne 2009), while other and more up-to-date models might yield different results. In conclusion, more research work about the plasma in the Suns Corona has to be made in order to find how much the true focal distance might be larger than just $550 \mathrm{AU}$.

\section{Beyond the Sun's focal sphere are the focal spheres of all planets}

Jupiter is the second larger mass in the solar system after the Sun, but in this focal game not only the mass matters: rather, what really matters is the ratio between the radius of the body squared and the mass of the body. For all planets, focal spheres have radiuses that can immediately calculated by virtue of the same equation used previously for the Sun. The results are given in Table 1 and Fig. 1 and show that all these radiuses range between 6,000 and 17,000 AU. In this regard, Jupiter qualifies as the second best choice for a space mission, requiring the spacecraft to reach 6,077 AU. Neptune qualifies third, with a focal sphere of 13,520 AU and Saturn comes fourth with a focal sphere of 14,420 AU. But the real surprise is the Earth, that qualifies just fifth with a focal sphere of 15,370 AU. And the Earth is indeed the best body we could use as a gravitational lens since we know about its atmosphere better than about any other planetary atmosphere. This is the "belt of focal spheres that we have so discovered and made known.

\section{References}

C. Maccone, 2009, in "Deep Space Flight and Communications Exploiting the Sun as a Gravitational Lens", published by Praxis-Springer, ISBN 978-3-540-72942-6. 
Detection and Dynamics of Transiting Exoplanets

Table 1.: Radii of the minimal focal spheres of the Naked Sun, Coronal Sun, and all planets.

\begin{tabular}{lccc}
\hline Body & $\begin{array}{c}\text { Radius } \\
{\left[10^{3} \mathrm{~km}\right]}\end{array}$ & $\begin{array}{c}\text { Mass } \\
{[\mathrm{kg}]}\end{array}$ & $\begin{array}{c}\text { Focal sphere radius } \\
{\left[10^{3} \mathrm{AU}\right]}\end{array}$ \\
\hline Naked Sun & 696.2 & $1.989 \times 10^{30}$ & 0.55 \\
Coronal Sun & $?$ & $1.989 \times 10^{30}$ & 1 \\
Jupiter & 71.59 & $1.898 \times 10^{27}$ & 6.1 \\
Neptune & 24.85 & $1.024 \times 10^{26}$ & 13.5 \\
Saturn & 60.35 & $5.684 \times 10^{26}$ & 14.4 \\
Earth & 6.387 & $5.974 \times 10^{24}$ & 15.4 \\
Uranus & 25.59 & $8.683 \times 10^{25}$ & 17.0 \\
Venus & 6.067 & $4.868 \times 10^{24}$ & 17.0 \\
Mars & 3.404 & $6.392 \times 10^{23}$ & 40.8 \\
Mercury & 2.446 & $3.285 \times 10^{23}$ & 41.0 \\
\hline \hline
\end{tabular}

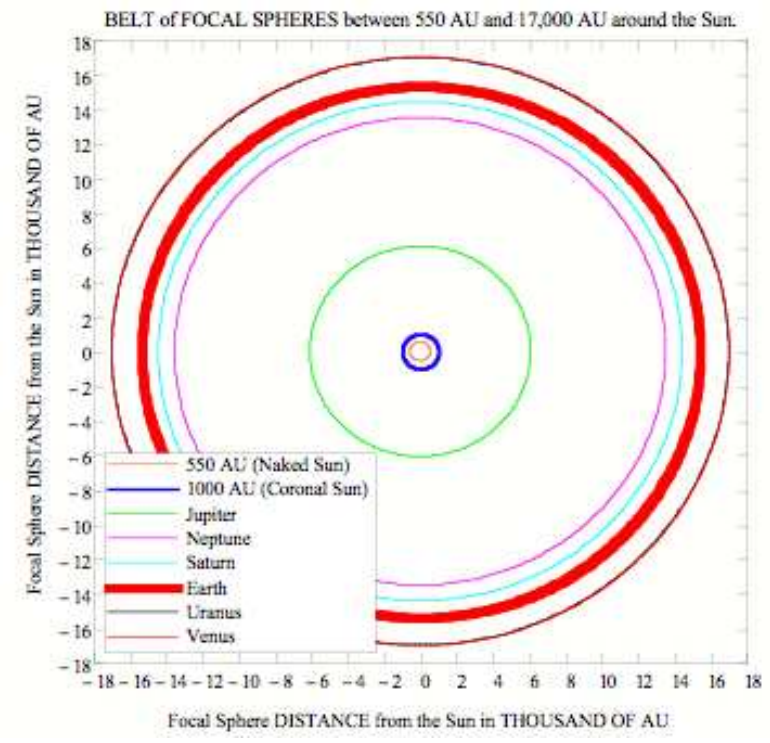

Figure 1.: The complete belt of focal spheres between 550 and 17,000 AU from the Sun, as created by the gravitational lensing effect of the sun and all planets, here shown to scale. 